\title{
Advances Toward Engineering Functionally Mature Human Pluripotent Stem Cell-Derived $\beta$ Cells
}

\author{
Leonardo Velazco-Cruz'1, Madeleine M. Goedegebuure ${ }^{1,2}$ and Jeffrey R. Millman 1,2* \\ ${ }^{1}$ Division of Endocrinology, Metabolism and Lipid Research, Washington University School of Medicine, St. Louis, MO, \\ United States, ${ }^{2}$ Department of Biomedical Engineering, Washington University in St. Louis, St. Louis, MO, United States
}

Human stem cell-derived $\beta$ (SC- $\beta$ ) cells have the potential to revolutionize diabetes treatment through disease modeling, drug screening, and cellular therapy. SC- $\beta$ cells are likely to represent an early clinical translation of differentiated human pluripotent stem cells (hPSC). In 2014, two groups generated the first in vitro-differentiated glucoseresponsive SC- $\beta$ cells, but their functional maturation at the time was low. This review will discuss recent advances in the engineering of SC- $\beta$ cells to understand and improve

\section{OPEN ACCESS}

Edited by:

Tiago G. Fernandes, University of Lisbon, Portugal

Reviewed by:

Xiaojun Lian,

Pennsylvania State University (PSU),

United States

Maria Cristina Nostro,

University Health Network (UHN),

Canada

*Correspondence: Jeffrey R. Millman

jmillman@wustl.edu

Specialty section:

This article was submitted to

Biomaterials,

a section of the journal Frontiers in Bioengineering and

Biotechnology

Received: 30 April 2020

Accepted: 22 June 2020

Published: 09 July 2020

Citation:

Velazco-Cruz L,

Goedegebuure MM and Millman JR (2020) Advances Toward Engineering

Functionally Mature Human

Pluripotent Stem Cell-Derived $\beta$ Cells.

Front. Bioeng. Biotechnol. 8:786.

doi: 10.3389/fbioe.2020.00786
SC- $\beta$ cell differentiation and functional maturation, particularly new differentiation strategies achieving dynamic glucose-responsive insulin secretion with rapid correction to normoglycemia when transplanted into diabetic mice.

Keywords: stem cells, diabetes, differentiation, pluripotent, transplantation

\section{INTRODUCTION}

Diabetes mellitus can be characterized as a disease of the $\beta$ cell, which results in improper insulin secretion and failure to maintain normal glycemia. Type 1 diabetes (T1D) is the result of the dysregulated autoimmune destruction of $\beta$ cells (Gillespie, 2006). Type 2 diabetes (T2D) is characterized by $\beta$ cell malfunction and depletion due to high stress caused by chronic hyperglycemia. Currently T1D and many T2D patients are reliant on exogenous insulin treatment. Exogenous insulin treatment requires constant monitoring and injections throughout the day, reducing quality of life and failing to accurately maintain normal glycemia leading to secondary complications (Caro et al., 2002; Powers and D'Alessio, 2011). Transplantation of whole pancreas or purified islets of Langerhans have been shown to result in exogenous insulin independence with accurate glycemic regulation in T1D and T2D patients (Posselt et al., 2010; Gruessner and Gruessner, 2016; Kandaswamy et al., 2018). Widespread application of islet transplantation is limited by replacement tissue availability and the need for immunosuppression (Millman and Pagliuca, 2017).

Human SC- $\beta$ cells are a promising alternative cell source for diabetes cell replacement therapy as well as disease modeling and drug screening (Millman and Pagliuca, 2017). In vitro differentiated SC- $\beta$ cells were first produced in 2014 (Pagliuca et al., 2014; Rezania et al., 2014). These early SC$\beta$ cells presented $\beta$ cell hallmarks, such as insulin secretion in response to glucose, expression of $\beta$ cell transcription factors, and in vivo function in weeks after transplantation in mice. However, critical differences remained between SC- $\beta$ cells and primary adult $\beta$ cells, including inferior insulin secretion per cell, dysregulated glucose-stimulated insulin secretion (GSIS) dynamics, and lower expression of key $\beta$ cell transcription factors. Recent studies, discussed here, have significantly advanced the functional maturity of SC- $\beta$ cells resulting in functionally enhanced SC- $\beta$ cells. These 
enhanced SC- $\beta$ cells have improved function, with some achieving dynamic insulin secretion marked by the presence of first and second phase insulin secretion. Despite the enhancement of these SC- $\beta$ cells, they fail to match the glucose responsiveness and transcriptomic profile of primary cadaveric islets (Baron et al., 2016; Nair et al., 2019; Velazco-Cruz et al., 2019; Hogrebe et al., 2020; Mahaddalkar et al., 2020). In this review we describe the advancements made for achieving enhanced SC- $\beta$ cells and the path toward differentiating fully functional SC- $\beta$ cells resembling cadaveric islets in terms of their function and transcriptomic profile.

\section{THE PATH TOWARD SC- $\beta$ CELLS}

The path toward differentiating SC- $\beta$ cells has proved challenging, having already spanned over two decades. Progress has occurred in waives as hard-fought milestones are achieved. Early pioneering work established methodologies for differentiating hPSCs toward definitive endoderm (D'Amour et al., 2005), the first developmental stage in the path toward making $\beta$ cells. Further sequential treatments of growth factors and small molecules continued to mimic pancreatic organogenesis guiding hPSCs through stages resembling definitive endoderm, gut-tube endoderm, pancreatic endoderm, and ultimately hormone expressing endoderm. The resulting insulin producing cells were polyhormonal, failed to maintain PDX1 and NKX6.1 expression, and were not glucose responsive (D'Amour et al., 2006). However, transplantation of hPSCderived pancreatic progenitors into immunocompromised mice allowed for their differentiation into monohormonal glucosestimulated insulin-secreting cells after several months in vivo (Kroon et al., 2008; Rezania et al., 2012). Since then, other groups have demonstrated that PDX1 and NKX6.1 expressing pancreatic progenitors have the potential of differentiating toward $\beta$ cells (Rezania et al., 2013; Schaffer et al., 2013; Nostro et al., 2015; Millman et al., 2016).

In 2014, two protocols were published for the efficient generation of glucose-responsive monohormonal in vitrodifferentiated SC- $\beta$ cells (Pagliuca et al., 2014; Rezania et al., 2014). These protocols generated pancreatic progenitors with high PDX1, NKX6.1 and low NGN3 expression. Low NGN3 expression through Vitamin $\mathrm{C}$ treatment distinguishes these pancreatic progenitors from earlier protocols (Kroon et al., 2008; Rezania et al., 2012). These pancreatic progenitors where then differentiated to hormone expressing endocrine cells through transient NGN3 upregulation by treatment with TGF $\beta$ R1 inhibitor ALK5i Type II (ALK5i) and thyroid hormone (T3). Air-liquid interface culture was observed to increase NGN3 expression relative to planar culture (Rezania et al., 2014) while the other protocol was completed in suspension culture (Pagliuca et al., 2014). The continued treatment of endocrine CHGA+ cells with ALK5i, T3, and $\gamma$-secretase (XXI or XX) drives the endocrine population toward monohormonal INS+ cells. The final stage of these protocols cultured the cells with ALK5i and T3 resulting in glucose responsive SC- $\beta$ cells making up $\sim 40 \%$ of the population. In addition to ALK5i and T3, Rezania includes compounds R428, an AXL inhibitor, with $\mathrm{N}$-acetyl cysteine, claiming them to drive expression of $\beta$ cell maturation gene MAFA. The Rezania et al. air-liquid interface culture format can be more easily replicated by laboratories with standard culture experience and equipment, however it is less scalable than the suspension culture format described in Pagliuca et al. which requires more specialized equipment and knowledge of 3D cell culture. These original SC- $\beta$ cells represented a monumental accomplishment being scalable, glucose responsive, transcriptionally similar to primary islets, and capable of regulating mouse blood glucose in weeks post transplantation. Despite these accomplishments the resulting SC- $\beta$ cells were functionally immature, secreting low levels of insulin, no dynamic insulin secretion, immature calcium response, and transcriptional differences remained relative to cadaveric islets with measurable differences in MAFA, UCN3, and GCK gene expression (Pagliuca et al., 2014; Rezania et al., 2014).

\section{ADVANCING SC- $\beta$ CELLS}

Early SC- $\beta$ cells are functionally immature lacking dynamic insulin secretion and observable functional maturation occurring after transplantation in vivo, marked by an increase in secreted insulin with time post transplantation (Pagliuca et al., 2014; Rezania et al., 2014; Russ et al., 2015). More functional SC- $\beta$ cells are needed to improve cell replacement outcomes and facilitate more robust disease modeling studies. Recent publications have demonstrated improved differentiation efficiency, higher glucose stimulated insulin secretion, first and second phase insulin secretion, response to multiple secretagogues, and fast in vivo glucose regulation upon transplantation (Table 1; Nair et al., 2019; Velazco-Cruz et al., 2019; Veres et al., 2019; Hogrebe et al., 2020; Mahaddalkar et al., 2020).

Velazco-Cruz et al. was first to report robust dynamic insulin secretion of SC- $\beta$ cells (Velazco-Cruz et al., 2019) with both first and second phase kinetics using a suspension differentiation protocol with temporal TGF $\beta$ modulation, cellular cluster size control, serum free media, endocrine enrichment without cell selection, and a simplified final stage media lacking notable prior factors (T3, N-acetyl cysteine, Trolox, and R428). The authors show that treatment with TGF $\beta$ R1 inhibitor, ALK5 inhibitor type II (ALK5i), is necessary for specification of the $\beta$ cell fate. However, upon $\beta$ cell specification permittance of TGF $\beta$ signaling is critical for SC- $\beta$ cell functional maturation. However, ALK5i is widely used in the final stage of many $\beta$ cell differentiation protocols (Pagliuca et al., 2014). The authors show that by re-sizing cellular clusters during the last stage of the differentiation, a process which involves partial dissociation of clusters as previously reported (Song and Millman, 2017), enhances static GSIS and nearly doubles the C-Peptide+ NKX6.1+ co-expressing SC- $\beta$ cell population. In dynamic secretion assays, robust dynamic function with a clear first phase, stable second phase, and 


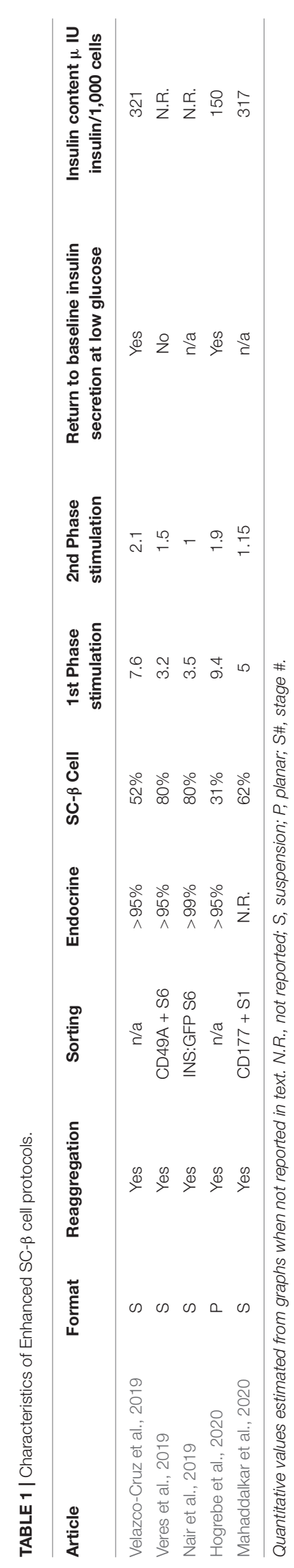

a return to basal levels after high glucose treatment ends was shown. However, a weakness of the study was that insulin secretion per cell and the degree insulin secretion was increased in response to high glucose varied across different hPSC lines. Transplantation of these cells improved glucose tolerance in mice.

Veres et al. employed some of the same changes as VelazcoCruz et al., including cellular cluster size control, serum free media, endocrine enrichment without cell selection, and a final stage media lacking T3, N-acetyl cysteine, Trolox, and R428 (Veres et al., 2019). Additionally, Veres et al. employs $\beta$ cell enrichment using cell sorting. Through a similar cellular reaggregation method, Veres et al. sees strong endocrine enrichment and an increase in the frequency of the C-Peptide+ /NKX6.1+ SC- $\beta$ cell population along with improved static GSIS. The authors further show that enrichment of the $\beta$ cell population through CD49a+ cell sorting improved static GSIS relative to unsorted and reaggregated SC- $\beta$ cells. CD49a+ sorted cells demonstrated first and second phase insulin secretion, however a return to basal levels did not occur when high glucose was removed. The authors did not show if reaggregated cells could undergo dynamic GSIS. The authors identify enterochromaffin-like cells in their SC$\beta$ cell preps and $\mathrm{CD} 49+$ sorting removes these cell types. Enterochromaffin cells, marked by TPH1 expression, secrete serotonin in the gut and are absent from the human pancreas. This study indicates $\beta$ cells and enterochromaffin cells share a similar developmental path resulting in their presence in $\beta$ cell differentiation protocols. It is unclear if the functional benefits observed by $\mathrm{CD} 49+$ sorting are due to the removal of off target cell types, such as enterochromaffin-like cells, or other mechanisms. Transplantation into mice was not investigated in this study.

In a separate study, Nair et al. achieved first phase insulin secretion using an insulin-driven GFP tag cell sorting approach and a reaggregation process (Nair et al., 2019). The authors achieved a high $\beta$ cell population with $80 \%$ C-Peptide/NKX6.1 co-expression. In functional studies the authors compare reaggregated GFP enriched cells to un-reaggregated unsorted $\beta$ cell clusters, with limited functional studies using reaggregated and unsorted clusters as a control. The authors see no first phase response in the immature clusters or the reaggregated and unsorted clusters while there GFP sorted reaggregated clusters have a first phase stimulation index of $\sim 3$ and no second phase response. However, the study design did not allow for distinguishing functional improvements related to endocrine enrichment by reaggregation or $\beta$ cell enrichment by sorting, like done with CD49a enrichment (Veres et al., 2019). This study was performed using only one insulin-driven GFP reporter cell line, making it unclear how well this approach would apply to other cell lines and whether the low levels of insulin secretion are due to the genetic engineering or genetic background of this cell line relative to other protocols. It is important to note that this differentiation protocol retains ALK5i and T3 during the final stage of differentiation, while other enhanced SC- $\beta$ cell protocols have removed them (RosadoOlivieri et al., 2019; Velazco-Cruz et al., 2019; Veres et al., 2019; 
Hogrebe et al., 2020; Maxwell et al., 2020). Therefore, it is possible that removal of these compound will allow for more robust dynamic secretion including the missing second phase observed by this protocol. Transplantation of these cells improved glucose tolerance in mice.

Hogrebe et al. (2020) used a different differentiation strategy for generating SC- $\beta$ cells, demonstrating that regulation of actin cytoskeleton polymerization controls differentiation to endocrine and other endodermal lineages. This insight led to development of a planar $\beta$ cell differentiation protocol. Other protocols use suspension (Pagliuca et al., 2014; Russ et al., 2015; RosadoOlivieri et al., 2019; Velazco-Cruz et al., 2019; Veres et al., 2019) or pseudo-suspension air-liquid interface (Rezania et al., 2014) culture methodologies for endocrine specification, increasing the technical expertise required for SC- $\beta$ cell differentiations. Using a novel planar differentiation protocol with actin depolymerizer latrunculin A driving endocrine specification, through neurogenin three upregulation, the authors generate SC$\beta$ cells which undergo dynamic GSIS. When these SC- $\beta$ cells are transplanted into mice, they rapidly reversed severe preexisting diabetes at a rate resembling that achieved by cadaveric islets.

In a controlled and parallel fashion, Hogrebe et al. compared his planar differentiated $\beta$ cells to suspension differentiated $\beta$ cells using the Velazco-Cruz et al. protocol. Using the HUES8 cell line, for which the two protocols were optimized, the suspension protocols had higher percent yields of CP+ NKX6.1+ SC- $\beta$ cells. Functionally, planar and suspension derived SC$\beta$ cells were similar when assayed by static and dynamic GSIS as well as insulin content. When assayed by real-time PCR, the planar and suspension derived SC- $\beta$ cells were similar. An equal number of planar and suspension derived SC- $\beta$ cells were transplanted into diabetic mice. Diabetes reversal with planar differentiated $\beta$ cells occurred in two weeks, while the suspension protocol took 5 weeks. This discrepancy in diabetes cure speed is interesting, as in vitro functional and transcriptomic assays did not show evident differences between the two protocols and based on reported differentiation efficiencies the suspension protocol generates a higher proportion of SC- $\beta$ cells. Single-cell RNA sequencing, comparing transcriptomes of planar and suspension derived SC- $\beta$ cells could reveal further insights into the source of this discrepancy. Importantly, the Hogrebe et al. planar protocol was able to successfully differentiate SC- $\beta$ cells from multiple pluripotent stem cell lines, with some cell lines matching cadaveric islets in function when assayed with dynamic perfusion assays. While the HUES8 suspension and planar derived SC$\beta$ cells were functionally similar, the planar protocol yielded higher functioning cells when applied to different cell lines. The robustness of the planar Hogrebe et al. differentiation protocol facilitates studies using more than one cell line (Maxwell et al., 2020; Velazco-Cruz et al., 2020).

Using a sorting approach for CD177/NB1 glycoprotein, Mahaddalkar et al. isolated anterior definitive endoderm cells with increased pancreatic and $\beta$ cell potential (Mahaddalkar et al., 2020). The authors characterize CD177+ cells to have increase PDX1 and NKX6.1 pancreatic progenitor potential when compared to unsorted and CD275+ definitive endoderm populations. Additionally, this work shows canonical WNT inhibition by IWP2 treatment to increase pancreatic progenitor differentiation efficiency, a finding supported by previous publications (Loh et al., 2014; Davenport et al., 2016; Zhu et al., 2016). Differentiation of CD177+ cells toward $\beta$ cells resulted in improved differentiation efficiency and function relative to unsorted cells. CD177+ cells presented a first phase insulin response with no second phase, while unsorted cells did not present a first or second phase insulin secretion (Mahaddalkar et al., 2020). The cells were not transplanted into mice.

Direct comparison of these studies is difficult, as assays evaluating function are variable, including technical methodologies, normalization strategies, and in vivo models differ. Normalizing SC- $\beta$ cells to cadaveric human islet insulin secretion is imperfect, as cadaveric islet function is highly variable within and between studies (Pagliuca et al., 2014; Nair et al., 2019; Velazco-Cruz et al., 2019; Veres et al., 2019). Standardized static and dynamic GSIS assays, normalized to DNA, can greatly facilitate comparison of differentiation protocols while imposing minimal burden on investigators. Standardization of in vivo assays are more challenging, as many mouse and diabetic models exist with variable severity of diabetes. By providing standardized in vitro GSIS results, individual researchers can better compare protocols and guide the differentiation protocols employed in their studies.

\section{FORGING A PATH FORWARD}

Despite advances, current SC- $\beta$ cells lack the functional maturity of cadaveric islets. In the continuing quest to functionally mature SC- $\beta$ cells, teams are employing novel technologies and approaches, such as single-cell sequencing, genetic engineering, cell sorting, and drug screening, to identify factors which contribute to $\beta$ cell differentiation and function. Recent publications have compared gene expression between adult and fetal or juvenile islets, with many adult genes having potential roles in the functional maturation of SC- $\beta$ cells. ERR $\gamma$ has been characterized to be enriched in adult vs neonatal mouse $\beta$ cells and mice deficient of $\beta$ cell ERR $\gamma$ fail to properly regulate their blood glucose (Yoshihara et al., 2016). Yoshira et al. differentiates hPSCs toward an immature $\beta$-like state in which many $\beta$ cells genes are expressed but are incapable of undergoing in vitro GSIS. The authors overexpress ERR $\gamma$ in these $\beta$-like cells and observed improvements to mitochondrial respiration and the $\beta$-like cells gain the ability to undergo in vitro GSIS. Upregulation of ERR $\gamma$ can potentially be used to further mature SC- $\beta$ cells. However, since its effects were only observed in immature insulin-expressing endocrine cells incapable of undergoing in vitro GSIS with immature mitochondrial respiration, it may not translate to more advanced protocols which are more metabolically mature (Nair et al., 2019). In a separate study, Arda et al. shows islet function increases in adult vs juvenile human islets identifying several genes associated 
with age in $\beta$ cells including ONECUT2, MAFA, TSHZ3, SIX2, and SIX3 (Arda et al., 2016). It remains to be investigated whether upregulation of these genes in SC- $\beta$ cells can drive their functional maturation.

Inhibition of YAP signaling has been shown to drive endocrine induction through neurogenin three upregulation and when incorporated into differentiation protocols during endocrine induction, using verteporfin, $\beta$ differentiation efficiency and function is enhanced (Rosado-Olivieri et al., 2019). Dynamic function is not assayed in this work. This work is supported by a previous study showing pancreatic progenitor endocrinogenesis is stimulated by YAP inhibition (Mamidi et al., 2018). Incorporation of YAP inhibitors during endocrine specification and YAP agonist during $\beta$ cell maturation may drive improvements to $\beta$ cell generation.

Recent work (Ameri et al., 2017; Cogger et al., 2017; Nair et al., 2019; Veres et al., 2019; Mahaddalkar et al., 2020) has shown that enrichment of certain cell populations can ultimately improve $\beta$ cell differentiation efficiency and function. Transcriptomic (Ameri et al., 2017) and proteomic (Cogger et al., 2017) approaches have revealed glycoprotein two as a cell surface marker beneficial for sorting PDX1+/NKX6.1+ pancreatic progenitors improving $\beta$ cell differentiation efficiency. Although functional improvements were not seen, this strategy may increase the proportion of $\beta$ cells generated using enhanced differentiation protocols. Enrichment of the $\beta$ cell population through sorting may enhance the functional maturation of SC$\beta$ cells (Nair et al., 2019; Veres et al., 2019). Whether this improvement is through cell-cell contact, paracrine signaling, or the removal of inhibitory cell types, such as enterochromaffin cells (Veres et al., 2019), remains to be determined with more robust studies necessary. Cell enrichment using cell sorting limits large scale production of SC- $\beta$ cells. However, the scale of production necessary for SC- $\beta$ cells may be compatible with magnetic-activated cell sorting approaches, particularly as they have proven beneficial. Additionally, identification of markers such as glycoprotein two can guide the search for small molecules to increase the population of cells expressing the desired markers. High throughput screening for small molecules affecting differentiation has been successful, with the identification of Rho-kinase inhibitor H1152, capable of increasing MAFA and UCN3 expression (Ghazizadeh et al., 2017).

Single-cell sequencing technologies are rapidly advancing our understanding of $\beta$ cell fate, differentiation, and functional maturation. Several studies have increasingly elucidated the $\beta$ cell transcriptome (Baron et al., 2016; Segerstolpe et al., 2016; Wang et al., 2016; Xin et al., 2016; Veres et al., 2019), revealing novel $\beta$ cell enriched genes which may be used as markers for driving $\beta$ cell functional maturation. Recently, single-cell patch-clamp sequencing was used linking $\beta$ cell gene expression to functional phenotypes revealing sets of genes correlating with $\beta$ cell function (Camunas-Soler et al., 2020). Veres et al. (2019) performed single-cell sequencing at multiple stages during SC- $\beta$ cell differentiations revealing transcriptomic profiles of each stage and genes whose expression is correlated to the acquisition of function by SC- $\beta$ cells. Epigenome analysis of SC- $\beta$ cell differentiation reveal LMX1B as a regulator of endocrine specification and circadian rhythms as a component toward SC- $\beta$ cell functional maturation (AlvarezDominguez et al., 2020). These studies give researchers a more accurate $\beta$ cell transcriptomic model to guide differentiation protocol development. To further advance SC- $\beta$ cell technology, researchers must continue efforts to build our understanding of $\beta$ cell development which guides development of $\beta$ cell differentiation protocols.

\section{DISCUSSION}

SC- $\beta$ cells are a promising cell source for diabetes cell therapy, disease modeling, and understanding human development. The use of small molecules and growth factors to drive stem cell differentiation toward $\beta$ cells, mimicking in vivo development, has proven a reliable strategy amenable to scaleup and genetic perturbations. Since the first fully in vitro differentiation protocols (Pagliuca et al., 2014; Rezania et al., 2014) capable of generating glucose responsive $\beta$ cells, the field has significantly advanced. Through the optimization of differentiation protocols, including the removal of ALK5i during the final stage of differentiation and reaggregation driven endocrine enrichment, enhanced SC- $\beta$ cells have greater glucose responsiveness undergoing dynamic GSIS. Currently, SC- $\beta$ cells are still less functional than cadaveric islets secreting lower amounts of insulin and a stable but lower in magnitude second phase insulin secretion (Nair et al., 2019; Velazco-Cruz et al., 2019; Veres et al., 2019). Transcriptionally, SC- $\beta$ cells resemble cadaveric islets more so than fetal islets, however critical differences remain, such as reduced expression of maturation factors MAFA and SIX3 (Veres et al., 2019). Using recently published technologies and approaches our understanding of $\beta$ cell development is improving, guiding the development of novel protocols capable of generating SC- $\beta$ cells with function matching that of primary islets. Generation of fully functionally mature SC- $\beta$ cells may be possible in the next few years and will drive diabetes cell therapies forward while providing a robust model for development and disease modeling.

\section{AUTHOR CONTRIBUTIONS}

LV-C and JM conceived the manuscript. All authors contributed to the article and approved the submitted version.

\section{FUNDING}

Support by the NIH (R01DK114233) and JDRF (5-CDA-2017391-A-N and 1-SRA-2020-928-S-B) was provided. 


\section{REFERENCES}

Alvarez-Dominguez, J. R., Donaghey, J., Rasouli, N., Kenty, J. H. R., Helman, A., Charlton, J., et al. (2020). Circadian entrainment triggers maturation of human in vitro islets. Cell Stem Cell 26, 108-122. doi: 10.1016/j.stem.2019.11.011

Ameri, J., Borup, R., Prawiro, C., Ramond, C., Schachter, K. A., Scharfmann, R., et al. (2017). Efficient generation of glucose-responsive beta cells from isolated GP2+ human pancreatic progenitors. Cell Rep. 19, 36-49. doi: 10.1016/j.celrep. 2017.03.032

Arda, H. E., Li, L., Tsai, J., Torre, E. A., Rosli, Y., Peiris, H., et al. (2016). Age-dependent pancreatic gene regulation reveals mechanisms governing human $\beta$ cell function. Cell Metab. 23, 909-920. doi: 10.1016/j.cmet.2016. 04.002

Baron, M., Veres, A., Wolock, S. L., Faust, A. L., Gaujoux, R., Vetere, A., et al. (2016). A single-cell transcriptomic map of the human and mouse pancreas reveals inter- and intra-cell population structure. Cell Syst. 3, 346-360. doi: 10.1016/j.cels.2016.08.011

Camunas-Soler, J., Dai, X.-Q., Hang, Y., Bautista, A., Lyon, J., Suzuki, K., et al. (2020). Patch-seq links single-cell transcriptomes to human islet dysfunction in diabetes. Cell Metab. 31, 1017-1031. doi: 10.1016/j.cmet.2020. 04.005

Caro, J. J., Ward, A. J., and O'Brien, J. A. (2002). Lifetime costs of complications resulting from type 2 diabetes in the U.S. Diabetes Care 25, 476-481. doi: 10.2337/diacare.25.3.476

Cogger, K. F., Sinha, A., Sarangi, F., McGaugh, E. C., Saunders, D., Dorrell, C., et al. (2017). Glycoprotein 2 is a specific cell surface marker of human pancreatic progenitors. Nat. Commun. 8:331. doi: 10.1038/s41467-017-00 561-0

D’Amour, K. A., Agulnick, A. D., Eliazer, S., Kelly, O. G., Kroon, E., and Baetge, E. E. (2005). Efficient differentiation of human embryonic stem cells to definitive endoderm. Cell 23, 1534-1541. doi: 10.1038/nbt 1163

D’Amour, K. A., Bang, A. G., Eliazer, S., Kelly, O. G., Agulnick, A. D., Smart, N. G., et al. (2006). Production of pancreatic hormone-expressing endocrine cells from human embryonic stem cells. Nat. Biotechnol. 24, 1392-1401. doi: $10.1038 /$ nbt1259

Davenport, C., Diekmann, U., Budde, I., Detering, N., and Naujok, O. (2016). Anterior-posterior patterning of definitive endoderm generated from human embryonic stem cells depends on the differential signaling of retinoic acid, Wnt-, and BMP-signaling. Stem Cells 34, 2635-2647. doi: 10.1002/stem. 2428

Ghazizadeh, Z., Kao, D.-I., Amin, S., Cook, B., Rao, S., Zhou, T., et al. (2017). ROCKII inhibition promotes the maturation of human pancreatic beta-like cells. Nat. Commun. 8:298. doi: 10.1038/s41467-017-00129-y

Gillespie, K. M. (2006). Type 1 diabetes: pathogenesis and prevention. Can. Med. Assoc. J. 175, 165-170. doi: 10.1503/cmaj.060244

Gruessner, A. C., and Gruessner, R. W. G. (2016). Long-term outcome after pancreas transplantation: a registry analysis. Curr. Opin. Organ Transplant. 21, 377-385. doi: 10.1097/MOT.0000000000000331

Hogrebe, N. J., Augsornworawat, P., Maxwell, K. G., Velazco-Cruz, L., and Millman, J. R. (2020). Targeting the cytoskeleton to direct pancreatic differentiation of human pluripotent stem cells. Nat. Biotechnol. 38, 460-470. doi: 10.1038/s41587-020-0430-6

Kandaswamy, R., Stock, P. G., Gustafson, S. K., Skeans, M. A., Curry, M. A., Prentice, M. A., et al. (2018). OPTN/SRTR 2016 annual data report: pancreas. Am. J. Transplant 18(Suppl. 1), 114-171. doi: 10.1111/ajt. 14558

Kroon, E., Martinson, L. A., Kadoya, K., Bang, A. G., Kelly, O. G., Eliazer, S., et al. (2008). Pancreatic endoderm derived from human embryonic stem cells generates glucose-responsive insulin-secreting cells in vivo. Nat. Biotechnol. 26, 443-452. doi: 10.1038/nbt1393

Loh, K. M., Ang, L. T., Zhang, J., Kumar, V., Ang, J., Auyeong, J. Q., et al. (2014). Efficient endoderm induction from human pluripotent stem cells by logically directing signals controlling lineage bifurcations. Cell Stem Cell 14, 237-252. doi: 10.1016/j.stem.2013.12.007

Mahaddalkar, P. U., Scheibner, K., Pfluger, S., Ansarullah, L., Sterr, M., Beckenbauer, J., et al. (2020). Generation of pancreatic $\beta$ cells from CD177+ anterior definitive endoderm. Nat. Biotechnol. doi: 10.1038/s41587-020-0492-5
Mamidi, A., Prawiro, C., Seymour, P. A., de Lichtenberg, K. H., Jackson, A., Serup, P., et al. (2018). Mechanosignalling via integrins directs fate decisions of pancreatic progenitors. Nature 564, 114-118. doi: 10.1038/s41586-018-0762-2

Maxwell, K. G., Augsornworawat, P., Velazco-Cruz, L., Kim, M. H., Asada, R., Hogrebe, N. J., et al. (2020). Gene-edited human stem cell-derived $\beta$ cells from a patient with monogenic diabetes reverse preexisting diabetes in mice. Sci. Transl. Med. 12:106. doi: 10.1126/scitranslmed.aax9106

Millman, J. R., and Pagliuca, F. W. (2017). Autologous pluripotent stem cellderived $\beta$-Like cells for diabetes cellular therapy. Diabetes 66, 1111-1120. doi: $10.2337 / \mathrm{db} 16-1406$

Millman, J. R., Xie, C., Van Dervort, A., Gürtler, M., Pagliuca, F. W., and Melton, D. A. (2016). Generation of stem cell-derived $\beta$-cells from patients with type 1 diabetes. Nat. Commun. 7:11463. doi: 10.1038/ncomms11463

Nair, G. G., Liu, J. S., Russ, H. A., Tran, S., Saxton, M. S., Chen, R., et al. (2019). Recapitulating endocrine cell clustering in culture promotes maturation of human stem-cell-derived $\beta$ cells. Nat. Cell Biol. 21, 263-274. doi: 10.1038/ s41556-018-0271-4

Nostro, M. C., Sarangi, F., Yang, C., Holland, A., Elefanty, A. G., Stanley, E. G., et al. (2015). Efficient generation of NKX6-1+ pancreatic progenitors from multiple human pluripotent stem cell lines. Stem Cell Rep. 4, 591-604. doi: 10.1016/j.stemcr.2015.02.017

Pagliuca, F. W., Millman, J. R., Gürtler, M., Segel, M., Van Dervort, A., Ryu, J. H., et al. (2014). Generation of functional human pancreatic $\beta$ cells in vitro. Cell 159, 428-439. doi: 10.1016/j.cell.2014.09.040

Posselt, A. M., Szot, G. L., Frassetto, L. A., Masharani, U., Tavakol, M., Amin, R., et al. (2010). Islet transplantation in type 1 diabetic patients using calcineurin inhibitor-free immunosuppressive protocols based on T-cell adhesion or costimulation blockade. Transplantation 90, 1595-1601. doi: 10.1097/TP. 0b013e3181fe1377

Powers, A., and D'Alessio, D. (2011). "Endocrine pancreas and pharmacotherapy of diabetes mellitus and hypoglycemia," in Goodman \& Gilman's The Pharmacological Basis of Therapeutics, eds L. L. Brunton, B. A. Chabner, and B. C. Knollmann (New York, NY: McGraw-Hill).

Rezania, A., Bruin, J. E., Arora, P., Rubin, A., Batushansky, I., Asadi, A., et al. (2014). Reversal of diabetes with insulin-producing cells derived in vitro from human pluripotent stem cells. Nat. Biotechnol. 32, 1121-1133. doi: 10.1038/nbt. 3033

Rezania, A., Bruin, J. E., Riedel, M. J., Mojibian, M., Asadi, A., Xu, J., et al. (2012). Maturation of human embryonic stem cell-derived pancreatic progenitors into functional islets capable of treating pre-existing diabetes in mice. Diabetes 61, 2016-2029. doi: 10.2337/db11-1711

Rezania, A., Bruin, J. E., Xu, J., Narayan, K., Fox, J. K., O’Neil, J. J., et al. (2013). Enrichment of human embryonic stem cell-derived NKX6.1-expressing pancreatic progenitor cells accelerates the maturation of insulin-secreting cells in vivo. Stem Cells 31, 2432-2442. doi: 10.1002/stem.1489

Rosado-Olivieri, E. A., Anderson, K., Kenty, J. H., and Melton, D. A. (2019). YAP inhibition enhances the differentiation of functional stem cell-derived insulinproducing beta cells. Nat. Commun. 10:1464. doi: 10.1038/s41467-019-094046

Russ, H. A., Parent, A. V., Ringler, J. J., Hennings, T. G., Nair, G. G., Shveygert, M., et al. (2015). Controlled induction of human pancreatic progenitors produces functional beta-like cells in vitro. EMBO J. 34, 1759-1772. doi: 10.15252/embj. 201591058

Schaffer, A. E., Taylor, B. L., Benthuysen, J. R., Liu, J., Thorel, F., Yuan, W., et al. (2013). Nkx6.1 controls a gene regulatory network required for establishing and maintaining pancreatic beta cell identity. PLoS Genet. 9:e1003274. doi: 10.1371/journal.pgen.1003274

Segerstolpe, A., Palasantza, A., Eliasson, P., Andersson, E.-M., Andréasson, A.-C., Sun, X., et al. (2016). Single-cell transcriptome profiling of human pancreatic islets in health and type 2 diabetes. Cell Metab. 24, 593-607. doi: 10.1016/j.cmet. 2016.08.020

Song, J., and Millman, J. R. (2017). Economic 3D-printing approach for transplantation of human stem cell-derived $\beta$-like cells. Biofabrication. 9:015002. doi: 10.1088/1758-5090/9/1/015002

Velazco-Cruz, L., Goedegebuure, M. M., Maxwell, K. G., Augsornworawat, P., Hogrebe, N. J., and Millman, J. R. (2020). SIX2 regulates human $\beta$ cell differentiation from stem cells and functional maturation in vitro. Cell Rep. 31:107687. doi: 10.1016/j.celrep.2020.107687 
Velazco-Cruz, L., Song, J., Maxwell, K. G., Goedegebuure, M. M., Augsornworawat, P., Hogrebe, N. J., et al. (2019). Acquisition of dynamic function in human stem cell-derived $\beta$ cells. Stem Cell Rep. 12, 351-365. doi: 10.1016/j.stemcr.2018. 12.012

Veres, A., Faust, A. L., Bushnell, H. L., Engquist, E. N., Kenty, J. H.R., Harb, G., et al. (2019). Charting cellular identity during human in vitro $\beta$-cell differentiation. Nature 569, 368-373. doi: 10.1038/s41586-019$1168-5$

Wang, Y. J., Schug, J., Won, K.-J., Liu, C., Naji, A., Avrahami, D., et al. (2016). Single-cell transcriptomics of the human endocrine pancreas. Diabetes 65, 3028-3038. doi: 10.2337/db16-0405

Xin, Y., Kim, J., Okamoto, H., Ni, M., Wei, Y., Adler, C., et al. (2016). RNA sequencing of single human islet cells reveals type 2 diabetes genes. Cell Metab. 24, 608-615. doi: 10.1016/j.cmet.2016.08.018

Yoshihara, E., Wei, Z., Lin, C. S., Fang, S., Ahmadian, M., Kida, Y., et al. (2016). $\operatorname{ERR} \gamma$ is required for the metabolic maturation of therapeutically functional glucose-responsive $\beta$ cells. Cell Metab. 23, 622-634. doi: 10.1016/j.cmet.2016. 03.005
Zhu, Z., Li, Q. V., Lee, K., Rosen, B. P., González, F., Soh, C. L., et al. (2016). Genome editing of lineage determinants in human pluripotent stem cells reveals mechanisms of pancreatic development and diabetes. Cell Stem Cell 18, 755-768. doi: 10.1016/j.stem.2016.03.015

Conflict of Interest: LV-C and JM are inventors in patents and patent applications relating to the differentiation of stem cell-derived $\beta$ cells.

The remaining author declares that the research was conducted in the absence of any commercial or financial relationships that could be construed as a potential conflict of interest.

Copyright ( 2020 Velazco-Cruz, Goedegebuure and Millman. This is an open-access article distributed under the terms of the Creative Commons Attribution License (CC BY). The use, distribution or reproduction in other forums is permitted, provided the original author(s) and the copyright owner(s) are credited and that the original publication in this journal is cited, in accordance with accepted academic practice. No use, distribution or reproduction is permitted which does not comply with these terms. 